\title{
Comparison of circular- and linear-stapled gastrojejunostomy in laparoscopic Roux-en-Y gastric bypass: a multicenter study
}

\author{
Piotr Major ${ }^{1,2}$, Michał R. Janik ${ }^{3}$, Michał Wysocki ${ }^{2,4}$, Maciej Walędziak ${ }^{3}$, Michał Pędziwiatr ${ }^{1,2}$, Piotr K. Kowalewski ${ }^{3}$, \\ Piotr Małczak ${ }^{1,2}$, Krzysztof Paśnik³ ${ }^{3}$ Andrzej Budzyński ${ }^{1,2}$ \\ ${ }^{1} 2^{\text {nd }}$ Department of General Surgery, Jagiellonian University Medical College, Krakow, Poland \\ ${ }^{2}$ Centre for Research, Training and Innovation in Surgery (CERTAIN Surgery), Krakow, Poland \\ ${ }^{3}$ Department of General, Oncological, Metabolic and Thoracic Surgery, Military Institute of Medicine, Warsaw, Poland \\ ${ }^{4}$ Students' Scientific Group at $2^{\text {nd }}$ Department of Surgery, Jagiellonian University Medical College, Krakow, Poland
}

Videosurgery Miniinv 2017; 12 (2): 140-146 DOI: https://doi.org/10.5114/wiitm.2017.66868

\begin{abstract}
Introduction: Laparoscopic Roux-en-Y gastric bypass (LRYGB) is a common, well-established procedure, but no consensus regarding selection of the gastrojejunostomy $(G J)$ technique has been reached, and standardization of this precise technique is far from being achieved.

Aim: To compare circular-stapled and linear-stapled GJ in $L R Y G B$ in terms of operative time and postoperative complications. Material and methods: This retrospective case-control study compared the perioperative and postoperative outcomes of $L R Y G B$ with a circular-stapled ( $L R Y G B-C S$ ) versus linear-stapled (LRYGB-LS) gastrojejunostomy. All patients, operated on in two academic referral care centers for bariatric surgery, were enrolled from April 2013 to June 2016. 457 patients were included (255 and 202 respectively in the LRYGB-CS and LRYGB-LS groups). After matching the groups for age, sex, body mass index, arterial hypertension, and presence of type 2 diabetes in a $1: 1$ ratio, 99 patients were enrolled in each.

Results: The total operative time was longer in the $L R Y G B-L S$ group (140 vs. $85 \mathrm{~min}, p<0.001)$. The postoperative hemorrhage and wound infection rates were lower in the LRYGB-LS group (2.1\% vs. $10.3 \%, p=0.021$, and $1.0 \% \mathrm{vs}$. $9.3 \%, p=0.011)$. The readmission rates were comparable ( $8.2 \%$ vs. $6.1 \%, p=0.593)$. There was no significant difference in the incidence of gastrojejunostomy leakage, stricture, port-site hernia, or marginal ulcer.

Conclusions: Both anastomosis types for $\angle R Y G B$ are safe and have low and comparable risks of postoperative complications. After $L R Y G B-C S$, postoperative bleeding and wound infections are slightly more frequent; however, the operative time is shorter.
\end{abstract}

Key words: obesity, bariatric surgery, laparoscopic Roux-en-Y gastric bypass, circular-stapled gastrojejunostomy, linear-stapled gastrojejunostomy.

\section{Introduction}

Laparoscopic Roux-en-Y gastric bypass (LRYGB) is a well-established procedure that has been performed in many countries for years, although considerable variability in the surgical technique has been noted [1-3]. Standardization of this precise technique is still far from being achieved. The various surgical gastrojejunostomy (GJ) techniques used during LRYGB are a good example of this variability. The most popular techniques are circular-stapled, linear-stapled, and hand-sewn anastomosis. The use

\section{Address for correspondence}

Maciej Walędziak MD, Department of General, Oncological, Metabolic and Thoracic Surgery, Military Institute of Medicine,

128 Szaserów St, 04-141 Warsaw, Poland, phone: +48 606387 636, e-mail: maciej.waledziak@gmail.com 
of circular-stapled GJ for LRYGB was first described in 1994 by Wittgrove et al. [4]. The use of a linear stapler when performing GJ during laparoscopic gastric bypass was then reported in two articles published in 2003: one by Korenkov et al. [5] and another by Olbers et al. [6]. Hand sewing is now infrequently performed because it is technically demanding and not reproducible. Circular- and linear-stapled GJ are widely accepted as faster and reproducible methods [7-10]. The two methods have been compared in numerous studies, but no consensus regarding which method is superior has yet been reached [3, 7-11]. Therefore, we designed the present multicenter study to compare GJ methods in two referral bariatric centers with respect to perioperative management and postoperative complications.

\section{Aim}

The aim of this study was to compare circular-stapled and linear-stapled GJ in LRYGB in terms of operative time and postoperative complications.

\section{Material and methods}

From April 2013 to June 2016, 475 morbidly obese patients underwent primary LRYGB in two academic referral bariatric centers. The first center performs LRYGB using only circular stapled gastroenterostomy, the second using only linear stapled gastroenterostomy. We collected data from the medical re- cords regarding the patients' baseline characteristics and postoperative complications. The institutional review board of each referral center approved this retrospective case-control study. The exclusion criteria were a history of previous weight loss surgery and a lack of necessary data. In total, 457 patients were enrolled and divided into 2 groups according to the $G J$ anastomosis technique: the circular stapler group (LRYGB-CS group, $n=255$ ) and the linear stapler group (LRYGB-LS group, $n=202$ ) (Figure 1). The baseline variables were age, sex, body mass index (BMI), preoperative weight loss, American Society of Anesthesiologists (ASA) score, and the following comorbidities: arterial hypertension, type 2 diabetes mellitus, obstructive sleep apnea, and dyslipidemia. Preoperative weight loss was defined as the difference between the initial $\mathrm{BMI}$ and the preoperative $\mathrm{BMI}$ and is expressed as preop $\Delta \mathrm{BMI}$.

\section{Surgical technique}

In the LRYGB-CS group, the stomach was transected using up to three linear staplers (EGIATRS60AMT, Endo GIA iDrive Ultra, Covidien, New Haven, $\mathrm{CT}$ ) creating a pouch with approximately $30 \mathrm{ml}$ volume capacity. After introducing the anvil (DST Series EEA OrVil, Covidien, New Haven, CT) transorally, ante-colic gastrojejunal anastomosis was performed using a $25 \mathrm{~mm}$ circular stapler (DST Series EEA XL25 open staple height $4.8 \mathrm{~mm}$; Covidien, New Haven, CT). Next using three linear staplers (Endo GIA iDrive

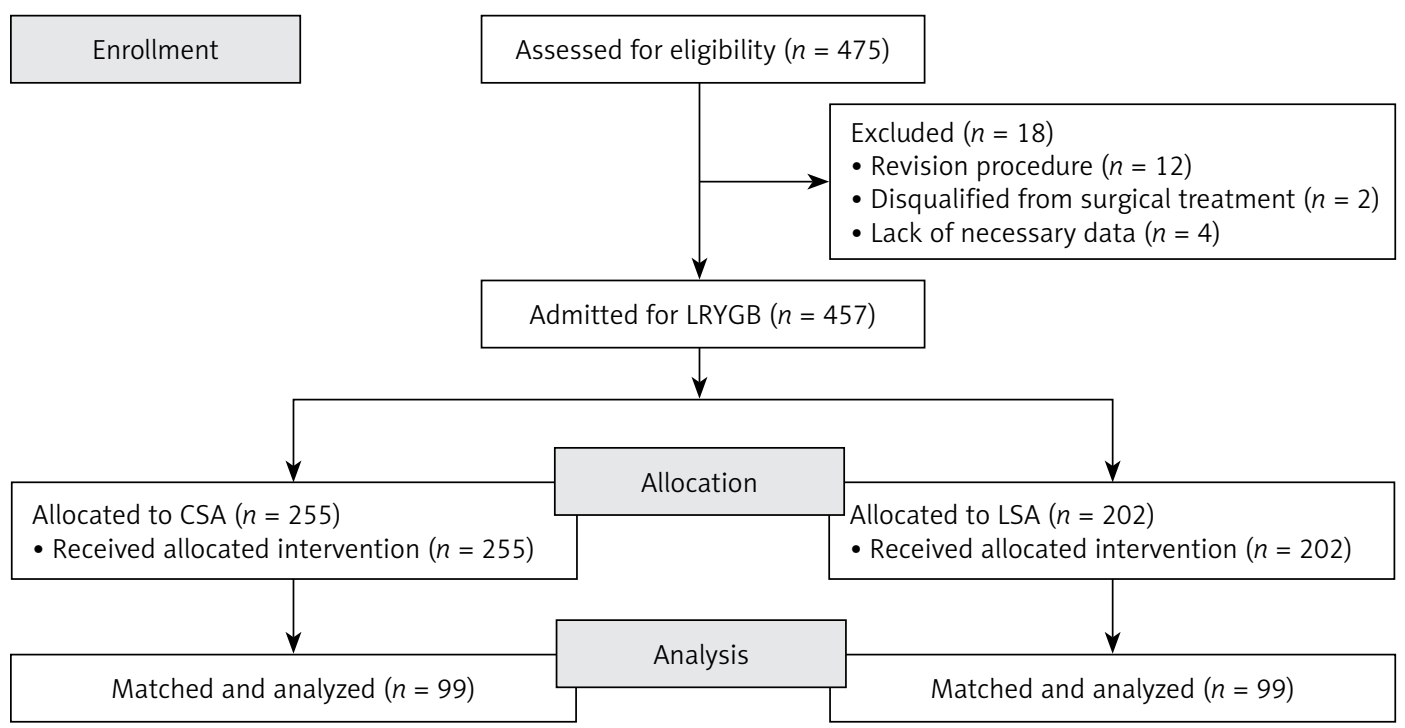

Figure 1. Study flowchart 
Ultra $60 \mathrm{~mm}$, with blue cartridge, Covidien, New Haven, $(T)$ the jejunojejunal anastomosis was made. In the LRYGB-LS group, the stomach was transected using up to three linear staplers (Ethicon Echelon EndoFlex, $45 \mathrm{~mm}$ with blue cartridges, open staple height $3.5 \mathrm{~mm}$, closed stapler height $1.5 \mathrm{~mm}$ ) then the Ethicon Echelon EndoFlex linear stapler $(45 \mathrm{~mm}$, with blue cartridges, open staple height $3.5 \mathrm{~mm}$, closed staple height $1.5 \mathrm{~mm}$ ) was used for ante-colic gastrojejunal anastomosis. The anterior wall defect was closed with 3/0 Vicryl (Ethicon) running suture. A linear stapler (Ethicon Echelon EndoFlex $45 \mathrm{~mm}$, with white cartridge, open staple height $2.5 \mathrm{~mm}$, closed staple height $1 \mathrm{~mm}$ ) was used for jejunojejunal anastomosis. No reinforcement stitches were used. Regardless of the GJ and jejunojejunal anastomosis techniques used, the lengths of the alimentary and enzymatic limbs were similar in all patients, respectively $150 \mathrm{~cm}$ and $100 \mathrm{~cm}$.

\section{Outcomes}

The medical records were evaluated for the operative time, length of hospital stay (LOS), 90-day readmission rate, and 90-day postoperative complication rate (complications included anastomotic leakage, postoperative hemorrhage, wound infection, portsite hernia, anastomotic stricture, and marginal ulcer). Anastomotic leakage was defined as leakage from the GJ diagnosed clinically and confirmed by computed tomography. Postoperative hemorrhage was defined as a significant drop in the hemoglobin level combined with either clinical signs of hemorrhage or the need for erythrocyte transfusion.

\section{Matching}

We performed matching because of heterogeneity between the two groups. The LRYGB-LS group was matched with the LRYGB-CS group in a $1: 1$ ratio by age ( \pm 4 years), sex, BMI $\left( \pm 2 \mathrm{~kg} / \mathrm{m}^{2}\right)$, presence of hypertension, and presence of type 2 diabetes mellitus. We used the algorithm described by Kawabata et al. [12] (1 : 1 matching procedure). Patients for whom we could not identify a suitable matching patient were excluded from the final analysis. The first analysis was performed using Statistica version 12.5. Matching and final analysis were performed using SAS software, University Edition (SAS Institute Inc., Cary, NC). Continuous outcomes of matched data were analyzed using the paired $t$-test or the
Wilcoxon signed ranks test. Dichotomous outcomes were analyzed using McNemar's test. Analysis of the matched (dependent) data differed from analysis of the unmatched (independent) data and was described in detail by Breslow and Day [13]. A $p$-value of $<0.05$ was considered statistically significant.

\section{Statistical analysis}

The patients' baseline data were compared using Student's $t$-test or the Mann-Whitney $U$ test for quantitative variables. Qualitative variables were compared using the $\chi^{2}$ test with or without Yates' correction.

\section{Results}

\section{Before matching}

The study flowchart is shown in Figure 1. In total, 457 patients were included in this study and allocated to either the LRYGB-LS $(n=255)$ or LRYGBCS group $(n=202)$. In the preliminary analysis, the groups were not comparable. The median age of patients in the LRYGB-LS group was 46 (39-53) years, and that of patients in the LRYGB-CS group was $41(35-48)$ years $(p<0.001)$. The median BMI in the LRYGB-LS group was 46.10 (41.80-51.90) $\mathrm{kg} / \mathrm{m}^{2}$, whereas that in the LRYGB-CS group was $42.24(39.52-44.98) \mathrm{kg} / \mathrm{m}^{2}(p<0.001)$. Patients in the LRYGB-LS group achieved greater preoperative weight loss than did patients in the LRYGB-CS group (preop $\Delta \mathrm{BMI}: 1.38$ (0.00-2.77) vs. 0.35 (0.00-2.57) $\mathrm{kg} / \mathrm{m}^{2}$, respectively; $p=0.009$ ). The LRYGB-LS group contained significantly fewer patients with arterial hypertension, dyslipidemia, type 2 diabetes mellitus, and obstructive sleep apnea (Table I). We therefore performed matching because of the heterogeneity between the two groups.

\section{After matching}

The median age of the patients in the LRYGB-LS group $(n=99)$ was $47(40-53)$ years, and that of the patients in the LRYGB-CS group $(n=99)$ was 48 $(41-53)$ years $(p=0.23)$. The median $\mathrm{BMI}$ in the LRYGB-LS group was $42.71(40.46-45.73) \mathrm{kg} / \mathrm{m}^{2}$, and that in the LRYGB-CS group was 42.45 (40.40$45.63) \mathrm{kg} / \mathrm{m}^{2}(p=0.16)$. There was a significantly greater prevalence of dyslipidemia in the LRYGB-LS group. The groups were comparable with respect to the other comorbidities (Table II). 
Table I. Baseline characteristics of the whole patient cohort and the two study groups

\begin{tabular}{|c|c|c|c|c|}
\hline Parameter & All patients $(N=457)$ & LRYGB-CS $(n=255)$ & LRYGB-LS $(n=202)$ & $P$-value \\
\hline Age [years] & $43(36-51)$ & $41(35-48)$ & $46(39-53)$ & $<0.001^{a}$ \\
\hline Female sex & $308(67)$ & $195(76)$ & $113(56)$ & \multirow[t]{2}{*}{$<0.001^{b}$} \\
\hline Male sex & $149(33)$ & $60(24)$ & $89(44)$ & \\
\hline Maximal preoperative BMI $\left[\mathrm{kg} / \mathrm{m}^{2}\right]$ & $44.06(40.91-48.98)$ & $42.24(39.52-44.98)$ & $48.15(43.42-53.76)$ & $<0.001^{c}$ \\
\hline BMI on day of operation $\left[\mathrm{kg} / \mathrm{m}^{2}\right]$ & $42.31(39.45-46.29)$ & $40.46(37.98-43.04)$ & $46.10(41.80-51.90)$ & $<0.001^{a}$ \\
\hline $\operatorname{Preop} \Delta \mathrm{BMI}\left[\mathrm{kg} / \mathrm{m}^{2}\right]$ & $0.74(0.00-2.61)$ & $0.35(0.00-2.57)$ & $1.38(0.00-2.77)$ & $0.009^{a}$ \\
\hline ASA score & 2 & 2 & 2 & $0.89^{a}$ \\
\hline Hypertension & $281(61.49)$ & $123(48.24)$ & $158(78.22)$ & $<0.001^{b}$ \\
\hline Diabetes mellitus type 2 & $157(34.35)$ & $52(20.39)$ & $105(51.98)$ & $<0.001^{b}$ \\
\hline Obstructive sleep apnea & $24(5.25)$ & $8(3.14)$ & $16(7.92)$ & $0.04^{c}$ \\
\hline
\end{tabular}

Data are presented as $n$ (\%) or median (interquartile range). ASA score is given as median. LRYGB-LS - laparoscopic Roux-en-Y gastric bypass with a linear-stapled gastrojejunostomy, LRYGB-CS - laparoscopic Roux-en-Y gastric bypass with a circular-stapled gastrojejunostomy, BMI - body mass index, ASA - American Society of Anesthesiologists, Preop $\triangle B M I$ - difference between initial BMI and preoperative BMI; 'Mann-Whitney test, ${ }^{b}$ Pearson's $\chi^{2}$ test, 'Fisher's exact test.

Table II. Baseline characteristics of the two study groups after $1: 1$ matching

\begin{tabular}{|lccc|}
\hline Parameter & LRYGB-LS $(n=99)$ & LRYGB-CS $(n=99)$ & $P$-value \\
\hline Age [years] & $47(40-53)$ & $48(41-53)$ & $0.23^{b}$ \\
\hline Female sex & $62(63)$ & $62(63)$ & - \\
\hline Preoperative BMI $\left[\mathrm{kg} / \mathrm{m}^{2}\right]$ & $42.71(40.46-45.73)$ & $42.45(40.40-45.63)$ & $0.16^{b}$ \\
\hline ASA score & 2 & 2 & $0.42^{\mathrm{b}}$ \\
\hline Dyslipidemia & $72(73.4)$ & $57(58.2)$ & $\mathbf{0 . 0 0 9}^{\mathrm{a}}$ \\
\hline Hypertension & $78(78.8)$ & $78(78.9)$ & - \\
\hline Diabetes mellitus type 2 & $49(49.5)$ & $49(49.5)$ & $0.78^{\mathrm{a}}$ \\
\hline Obstructive sleep apnea & $7(7.1)$ & $6(6.1)$ & - \\
\hline
\end{tabular}

Data are presented as $n$ (\%) or median (interquartile range). ASA score is given as median. $L R Y G B$-LS-laparoscopic Roux-en-Y gastric bypass with a linear-stapled gastrojejunostomy, LRYGB-CS - laparoscopic Roux-en-Y gastric bypass with a circular-stapled gastrojejunostomy, BMI-body mass index, ASA - American Society of Anesthesiologists; ${ }^{a}$ McNemar's test, ${ }^{b}$ Signed rank test.

The total operative time was significantly longer in the LRYGB-LS group (140 (100-180) vs. 85 (70-115) $\mathrm{min}, p<0.001)$. The rate of postoperative hemorrhage was significantly lower in the LRYGB-LS than the LRYGB-CS group ( $2.1 \%$ vs. $10.3 \%, p=0.02$ ). The mean length of hospital stay was significantly shorter in the LRYGB-LS group (3 (2-4) vs. 5 (3-5) days, $p<0.001)$. One of the hospitals which were doing only LRYGB using linear stapled gastroenterostomy precedes the ERAS-based perioperative protocol. There was a lower rate of wound infection in the LRYGB-LS than the LRYGB-CS group (1.0\% vs. 9.3\%, $p=0.01)$. The readmission rate was comparable between the two groups ( $8.2 \%$ vs. $6.1 \%, p=0.59$ ). There was no significant difference in the incidence of GJ anastomotic leakage, GJ anastomotic stricture, port-site hernia, or marginal ulcer (Table III).

\section{Discussion}

The LRYGB has become one of the most popular bariatric procedures [1,14-16]. However, there is currently no standard technique for the GJ anastomosis in LRYGB. Analysis of the postoperative course is difficult and numerous factors can influence surgical outcomes. One of these factors is the surgical 
Table III. Comparison between the two study groups after $1: 1$ matching

\begin{tabular}{|lccc|}
\hline Parameter & LRYGB-LS $(n=99)$ & LRYGB-CS $(n=99)$ & P-value \\
\hline Length of hospital stay, median (IQR) [days] & $3(2-4)$ & $5(3-5)$ & $<0.001^{\mathrm{b}}$ \\
\hline Operative time, median (IQR) [min] & $140(100-180)$ & $85(70-115)$ & $<0.001^{\mathrm{b}}$ \\
\hline Anastomotic leakage, $n(\%)$ & $1(1.0)$ & $1(1.0)$ & $1.00^{\mathrm{a}}$ \\
\hline Postoperative hemorrhage, $n(\%)$ & $2(2.1)$ & $10(10.3)$ & $0.02^{\mathrm{a}}$ \\
\hline Wound infection, $n(\%)$ & $1(1.0)$ & $9(9.3)$ & $0.01^{\mathrm{a}}$ \\
\hline Port site hernia, $n(\%)$ & $4(4.1)$ & $1(1.0)$ & $0.18^{\mathrm{a}}$ \\
\hline Anastomotic stricture, $n(\%)$ & $1(1.0)$ & $1(1.0)$ & $1.00^{\mathrm{a}}$ \\
\hline Marginal ulcer, $n(\%)$ & $1(1.0)$ & $6(6.1)$ & $1.00^{\mathrm{a}}$ \\
\hline Readmissions, $n(\%)$ & $8(8.2)$ & $0(0)$ & $0.59^{\mathrm{a}}$ \\
\hline Fatal cases, $n(\%)$ & $1(1.0)$ & - \\
\hline
\end{tabular}

Data are presented as $n$ (\%) or median (interquartile range). LRYGB-LS - laparoscopic Roux-en-Y gastric bypass with a linear-stapled gastrojejunostomy, LRYGB-CS - laparoscopic Roux-en-Y gastric bypass with a circular-stapled gastrojejunostomy; ${ }^{a}$ McNemar's test, ${ }^{b}$ Signed rank test.

technique, but it is not the only important element [17-21].

Shope et al. [20] performed one of the earliest studies in this field. They compared circular- and linear-stapled GJ in a group of 61 patients and reported that the GJ anastomosis technique may be based on the operating surgeon's preference. Only the operative time was shorter in the LRYGB-LS group. Although the operative time in most studies was shorter when using the linear stapler for GJ [3, 22-24], the operative time in the present study was significantly shorter in the LRYGB-CS group.

The most common complication in our cohort was postoperative hemorrhage (5.71\%). In the present study, the use of a circular stapler significantly increased the risk of postoperative hemorrhage, which has also been commonly reported in other studies. In a meta-analysis of five studies by Penna et al. [10], the use of a circular stapler was associated with a $117 \%$ greater risk of postoperative bleeding in the pooled analysis (pooled odds ratio $(\mathrm{OR})=2.17$; 95\% confidence interval $(\mathrm{Cl}): 1.49-$ 3.23). An increased risk of postoperative bleeding was also reported in a more recent study by Edholm and Sundbom [3] (OR = 1.9; 95\% Cl: 1.2-2.9). This finding is comparable between the studies by Edholm and Sundbom [3] (2.03\%) and Finks et al. [24] (2.3\%). Considering all cases, before matching, postoperative hemorrhage was diagnosed significantly more often in the LRYGB-CS group (9.06\% vs. 1.98\%, $p=0.001)$. In the LRYGB-LS group, from 2 cases with postoperative hemorrhage, 1 was intraluminal and 1 intra-abdominal. In the LRYGB-CS group, from 23 cases with hemorrhage, 17 were intraluminal and 6 intra-abdominal.

The wound infection rates in the present study were comparable to those in studies by Finks et al. [24] (3.20\%) and Bendewald et al. [11] (2.91\%). The risk of infectious wound complications was significantly higher in the studies by Penna et al. [10] (pooled OR $=3.13 ; 95 \% \mathrm{Cl}: 2.27-4.35$ ) and Edholm and Sundbom [3] (OR = 9.7; 95\% Cl: 6.8-13.9). We also revealed that the risk of wound infection increased with the use of a circular stapler. In the LRYGBCS group, the stapling device was introduced directly through the wound, and in the LRYGB-LS group, the stapling device was inserted through the laparoscopic trocars. This may explain the differences.

The most common late postoperative complication in the present study was port-site hernia. The type of stapler used did not significantly change the risk of port-site hernia. However, we found no reference in the literature for comparison.

In the current study, the incidence of anastomotic leakage was not significantly influenced by the use of a circular versus linear stapler. In the study by Edholm and Sundbom [3], patients in the LRYGB-CS group had a greater risk of anastomotic leakage than did patients in the LRYGB-LS group (OR $=2.8 ; 95 \%$ $\mathrm{Cl}: 1.5-5.0)$. In the previously published meta-analysis by Penna et al. [10], there was no significant difference in the anastomotic leakage rate between the 
two groups (pooled $\mathrm{OR}=0.72 ; 95 \% \mathrm{Cl}: 0.37-1.37$ ). In contrast, Bendewald et al. [11] reported that the LRYGB-CS group had a lower rate of leakage than did the LRYGB-LS group (3.6\% vs. $8.0 \%$ ).

In a comparison of all three techniques, Lee et al. [25] stated that considering the comparable stricture rates and weight loss effects, surgeons should use the technique that best matches their surgical skill level. Previous studies have demonstrated that the risk of anastomotic stricture is greater when using a circular stapler for GJ (pooled OR = 3.33; 95\% Cl: 1.14-10.0). Qureshi et al. [26] recently reported a significantly higher $\mathrm{GJ}$ stricture rate in the LRYGBLS than the LRYGB-CS group (4.42\% vs. $1.18 \%$ ). In the present study in groups before matching, the stricture rate in the LRYGB-CS group was $2.36 \%$ and that in the LRYGB-LS group was $0.5 \%$, and the risk of anastomotic stricture was not affected by the stapling technique (OR $=2.25 ; 95 \% \mathrm{Cl}: 0.24-20.95)$. After matching, the stricture rate was the same (1\%).

In the study by Edholm and Sundbom [3], the OR for marginal ulceration was significantly increased by the use of a circular stapler $(\mathrm{OR}=3.1 ; 95 \% \mathrm{Cl}$ : 1.8-5.3). In the studies by Leyba et al. [23], Bendewald et al. [11], and Finks et al. [24] as well as in the present study, the OR of marginal ulceration was also not associated with the stapler type $(O R=2.40$; 95\% Cl: 0.25-23.41).

A shorter length of stay was observed in a center where the protocol of Enhanced Recovery After Surgery (ERAS) is used routinely. Numerous studies have demonstrated that application of the ERAS protocol is associated with significant shortening of LOS [27].

At the end of the study we decided to analyze the cost-effectiveness of each method. Total cost of staplers in LRYGB-LS was about $20 \%$ lower. Average cost of staplers was 1050 USD in the LRYGB-LS group and 1300 USD in the LRYGB-CS group.

Despite the fact that this study was conducted in two different bariatric centers, both GJ techniques were relatively safe for the patients and had comparable complication rates. Surgeons should be aware of the higher rates of postoperative bleeding and wound complications when performing LRYGB-CS. Further studies are needed, especially to determine the influence of the surgical technique on late postoperative complications and hospital readmission.

The limitations of the present study are its nonrandomized design and the relatively small sample of patients. The low number of subjects results in low power of the study. Thus, the risk of type 2 error is large. To increase precision and power we performed matching. The LRYGB procedures were performed at two different bariatric centers, and the study groups were demographically heterogeneous and differed in their patient-dependent preoperative factors. To overcome this limitation, we performed matching to obtain the most comparable groups. Additionally, the present study lacked data on postoperative internal hernia.

\section{Conclusions}

The present results suggest that both circularand linear-stapled GJ anastomoses for LRYGB are safe and have low and comparable risks of postoperative complications. Postoperative bleeding and wound infections are slightly more frequent with circular stapling; however, the operative time is significantly shorter.

\section{Conflict of interest}

The authors declare no conflict of interest.

\section{References}

1. Buchwald H, Oien DM. Metabolic/bariatric surgery worldwide 2011. Obes Surg 2013; 23: 427-36.

2. Nguyen NT, Goldman C, Rosenquist CJ, et al. Laparoscopic versus open gastric bypass: a randomized study of outcomes, quality of life, and costs. Ann Surg 2001; 234: 279-89; discussion 289-91.

3. Edholm D, Sundbom M. Comparison between circular- and linear-stapled gastrojejunostomy in laparoscopic Roux-en-Y gastric bypass: a cohort from the Scandinavian Obesity Registry. Surg Obes Relat Dis 2015; 11: 1233-6.

4. Wittgrove AC, Clark GW, Tremblay LJ. Laparoscopic gastric bypass, Roux-en-Y: preliminary report of five cases. Obes Surg 1994; 4: 353-7.

5. Korenkov M, Goh P, Yücel N, Troidl H. Laparoscopic gastric bypass for morbid obesity with linear gastroenterostomy. Obes Surg 2003; 13: 360-3.

6. Olbers T, Lönroth H, Fagevik-Olsén M, Lundell L. Laparoscopic gastric bypass: development of technique, respiratory function, and long-term outcome. Obes Surg 2003; 13: 364-70.

7. Janik MR, Rogula T, Bielecka I, et al. Quality of life and bariatric surgery: cross-sectional study and analysis of factors influencing outcome. Obes Surg 2016; 26: 2849-55.

8. Giordano S, Salminen P, Biancari F, Victorzon M. Linear stapler technique may be safer than circular in gastrojejunal anastomosis for laparoscopic Roux-en-Y gastric bypass: a meta-analysis of comparative studies. Obes Surg 2011; 21: 1958-64.

9. Jiang HP, Lin LL, Jiang X, Qiao HQ. Meta-analysis of hand-sewn versus mechanical gastrojejunal anastomosis during laparo- 
scopic Roux-en-Y gastric bypass for morbid obesity. Int J Surg 2016; 32: 150-7.

10. Penna M, Markar SR, Venkat-Raman V, et al. Linear-stapled versus circular-stapled laparoscopic gastrojejunal anastomosis in morbid obesity: meta-analysis. Surg Laparosc Endosc Percutan Tech 2012; 22: 95-101.

11. Bendewald FP, Choi JN, Blythe LS, et al. Comparison of handsewn, linear-stapled, and circular-stapled gastrojejunostomy in laparoscopic Roux-en-Y gastric bypass. Obes Surg 2011; 21: 1671-5.

12. Kawabata H, Tran M, Hine P. Using SAS ${ }^{\circledR}$ to Match Cases for Case Control Studies. Sugi 29. Montreal, Canada 2004; 173-29.

13. Breslow NE, Day NE. Statistical methods in cancer research. Vol. I. The analysis of case-control studies. IARC Sci Publ 1980; 5-338.

14. Picot J, Jones J, Colquitt JL, et al. The clinical effectiveness and cost-effectiveness of bariatric (weight loss) surgery for obesity: a systematic review and economic evaluation. Health Technol Assess 2009; 13: 1-190, 215-357, iii-iv.

15. O'Brien PE, MacDonald L, Anderson M, et al. Long-term outcomes after bariatric surgery: fifteen-year follow-up of adjustable gastric banding and a systematic review of the bariatric surgical literature. Ann Surg 2013; 257: 87-94.

16. Janik MR, Edward S, Paśnik K. Present status of bariatric surgery in Poland. Videosurgery Miniinv 2016; 11: 22-5.

17. Sauerland S, Angrisani L, Belachew M, et al. Obesity surgery: evidence-based guidelines of the European Association for Endoscopic Surgery (EAES). Surg Endosc 2005; 19: 200-21.

18. Matłok M, Pędziwiatr M, Major P, et al. One hundred seventynine consecutive bariatric operations after introduction of protocol inspired by the principles of enhanced recovery after surgery $\left(\right.$ ERAS $\left.^{\oplus}\right)$ in bariatric surgery. Med Sci Monit 2015; 21: 791-7.

19. Thorell A, MacCormick AD, Awad S, et al. Guidelines for perioperative care in bariatric surgery: enhanced recovery after surgery (ERAS) society recommendations. World I Surg 2016; 40: 2065-83.

20. Shope TR, Cooney RN, McLeod J, et al. Early results after laparoscopic gastric bypass: EEA vs GIA stapled gastrojejunal anastomosis. Obes Surg 2003; 13: 355-9.

21. Kwiatkowski A, Janik MR, Paśnik K, Stanowski E. The effect of oversewing the staple line in laparoscopic sleeve gastrectomy: randomized control trial. Videosurgery Miniinv 2016; 11: 149-55.

22. Binda A, Jaworski P, Kudlicka E, et al. The impact of selected factors on parameters of weight loss after sleeve gastrectomy. Videosurgery Miniinv 2016; 11: 288-94.

23. Leyba JL, Llopis SN, Isaac J, et al. Laparoscopic gastric bypass for morbid obesity - a randomized controlled trial comparing two gastrojejunal anastomosis techniques. JSLS 2008; 12: 385-8.

24. Finks JF, Carlin A, Share D, et al. Effect of surgical techniques on clinical outcomes after laparoscopic gastric bypass: results from the Michigan Bariatric Surgery Collaborative. Surg Obes Relat Dis 2011; 7: 284-9.

25. Lee S, Davies AR, Bahal S, et al. Comparison of gastrojejunal anastomosis techniques in laparoscopic Roux-en-Y gastric bypass: gastrojejunal stricture rate and effect on subsequent weight loss. Obes Surg 2014; 24: 1425-9.
26. Qureshi A, Podolsky D, Cumella L, et al. Comparison of stricture rates using three different gastrojejunostomy anastomotic techniques in laparoscopic Roux-en-Y gastric bypass. Surg Endosc 2015; 29: 1737-40.

27. Małczak P, Pisarska M, Piotr M, et al. Enhanced recovery after bariatric surgery: systematic review and meta-analysis. Obes Surg 2017; 27: 226-35.

Received: 16.01.2017, accepted: 15.03.2017. 